\title{
Supporting Information - The Impact of Nanostructuring on the Phase Behavior of Insertion Materials: The Hydrogenation Kinetics of a Magnesium Thin Film
}

Lars J. Bannenberg, ${ }^{*}{ }^{\dagger}$ Herman Schreuders, ${ }^{\ddagger}$ Lambert van Eijck, ${ }^{\dagger}$ Jouke R. Heringa, ${ }^{\dagger}$ Nina-Juliane Steinke, ${ }^{\S}$ Robert Dalgliesh, ${ }^{\S}$ Bernard Dam, ${ }^{\ddagger}$ Fokko M. Mulder, ${ }^{\ddagger}$ Ad A. van Well ${ }^{\dagger}$

† Department of Radiation Science \& Technology, Delft University of Technology, Mekelweg 15, Delft, 2629 JB, The Netherlands

$\ddagger$ Department of Chemical Engineering, Delft University of Technology, Julianalaan 134, Delft, 2628 BL, The Netherlands

§ ISIS, Rutherford Appleton Laboratory, OXı1 oQX, Didcot, Oxfordshire, United Kingdom

\section{Sample and sample environment}

Samples are produced by sputtering layers on a 3-inchdiameter circular fused quartz substrate with a density of $\rho=2.3 \mathrm{gcm}^{-3}$ using magnetron sputter deposition in a vacuum chamber. More details about the sample preparation can be found in Mooij and Dam. ${ }^{23,24}$

The sample is hydrogenated inside a tailor made hydrogenation cell, with controlled pressure, flow and temperature. As a loading gas, a mixture of $99.90 \%$ argon and $0.10 \% \mathrm{D}_{2}$ is used. During the experiments, a constant flow of $10 \mathrm{sccm}$ was maintained and the temperature was kept constant at $T=80^{\circ}$. The cell is equipped with thin aluminium windows at the entrance and exit to allow neutrons to pass. A tungsten halogen white light source with a peak intensity at a wavelength of $600 \mathrm{~nm}$ is mounted on the bottom, and a 1/2.5" Aptina CMOS 2592x1944 pixel monochrome camera with an Edmunds Optics 55-906 lens is installed on the top for the optical transmission measurements. The camera props a circular area with a diameter of $5 \mathrm{~mm}$.

\section{NR Measurements}

Neutron Reflectometry measurements are performed at Offspec, ISIS, Rutherford Appleton Laboratory. ${ }^{32}$ Static measurement are conducted at every converged state using both an incident angle of 8.7 and 34.7 mrad resulting in a $Q$-range of $0.08-1.4 \mathrm{~nm}^{-1}$ with a wave-vector transfer resolution of $\Delta Q / Q=0.02$.

The kinetic neutron reflectometry measurements are conducted at Offspec using event-mode data acquisition with an incident angle of $12 \mathrm{mrad}$ and a resulting $Q$-range of $0.08-0.9 \mathrm{~nm}^{-1}$. A fixed slit close to the sample in combination with the opening of the other slits is used to increase intensity with a factor of approx. three at the cost of a lower resolution of $\Delta Q / Q=0.06$.

\section{NR Data Processing and Fitting}

The neutron reflectometry data are fitted using STAR to obtain values for the layer thickness, roughness and Scattering Length Density (SLD) for each layer. ${ }^{33}$ The eventmode data is time sliced with various bin sizes: for the loading 1 minute bins for the first 10 minutes, 10 minute bins for the subsequent 110 minutes and 30 minute bins for the remaining time. For the unloading, we used 10 minute slices for the first $120 \mathrm{~min}$ and $30 \mathrm{~min}$ slices afterwards, this being a compromise between a stable, 'physical' state and counting statistics. To reduce the number of fitting parameters, we kept the palladium layer thickness and the SLD of the substrate and the Pd layer constant throughout all our fits reported. SLD profiles of selected measurements during loading and unloading are displayed in Figure $S_{1}$ and the corresponding parameters are given in Table S1. The scattering length densities of the layers of the virgin sample was within $10 \%$ of their respective bulk values. Note that as a result of the limited Q-range it is not possible to get an accurate estimation of the layers roughnesses.

An additional thin depletion layer between the lower titanium layer and the substrate is used to account for a locally lower titanium density/deuterium loading. This layer shows up as a steep 'dip' in the SLD-profiles of Figure S1. This locally lower SLD is likely a result of a poor adhesion of titanium to the substrate that leads to a lower $\mathrm{Ti}$ density and hence also a lower $\mathrm{TiD}_{\mathrm{x}}$ density and/or to a slightly lower degree of deuteration of the Ti layer near the substrate.

The depletion layer can only be detected if the contrast between the lower $\mathrm{Ti}$ layer and the substrate is low, i.e. if 

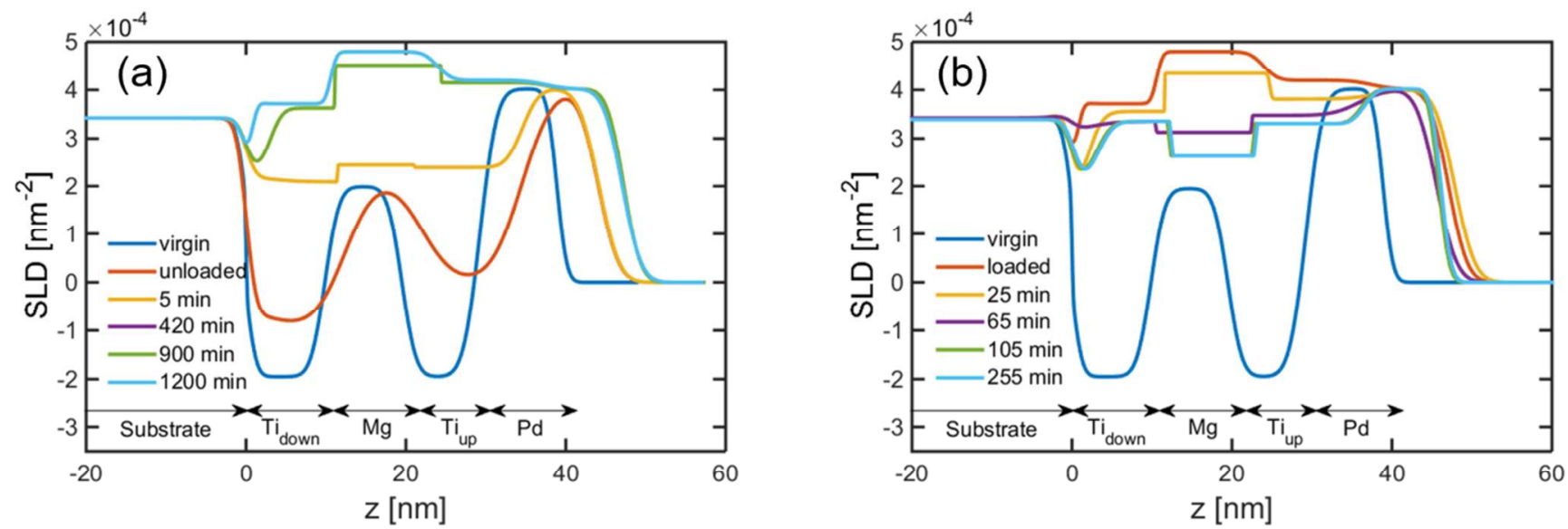

Fig. S1 Scattering Length Profiles (SLD) at selected moments during (a) loading and (b) unloading. $z$ refers to the out of plane coordinate of the sample and the origin is chosen at the substrate surface.
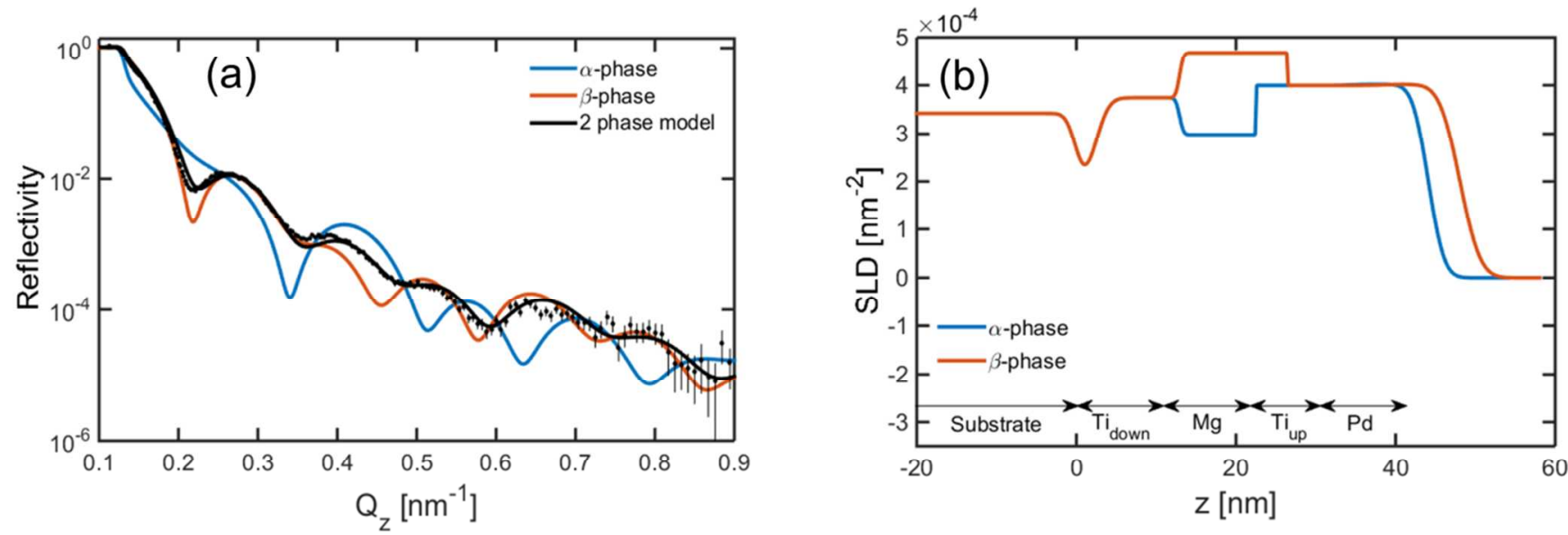

Fig. S2. Illustration of the 2-phase model that is used to fit the NR data of time slice $t=780-810$ min where the fraction of $\beta-\mathrm{MgD}_{2-\mathrm{y}}$ is equal to $f=0.78$. (a) Fitted reflectogram for the contribution for $\alpha-\mathrm{MgD}_{\mathrm{x}}, \beta-\mathrm{MgD}_{2-\mathrm{y}}$ and the resulting weighted fit. (b) SLD profiles for the $\alpha-\mathrm{MgD}_{\mathrm{x}}$ and $\beta-\mathrm{MgD}_{2-\mathrm{y}}$ layer.

$\mathrm{SLD}_{\mathrm{Ti}} \approx \mathrm{SLD}_{\mathrm{SiO}_{2}}$. In other case, the interface roughness is sufficient to account for the locally lower SLD.

To compute the deuterium content of the $\mathrm{Mg}$ layer from the fitted layer's SLD, we make use of the fact that the number of $\mathrm{Mg} / \mathrm{Ti}$ atoms inside a layer remains constant, and by using the layer thickness and scattering length density of the virgin sample. The scattering length density for a $\mathrm{Mg}$ layer is given by

$$
S L D_{M g D_{x}}=N_{M g D_{x}}\left(b_{M g}+x b_{D}\right) \text {, }
$$

where $N$ denotes the atomic number density, $b_{i}$ the scattering length of isotope $i$ and $x$ is equal to the ratio of $\mathrm{D}$ to $\mathrm{Mg}$ atoms in the layer. The isotope-dependent scattering length is a well-known quantity that is tabulated in literature. Hence, by knowing the SLD of the virgin Mg layer $(x=0)$, one can compute the atomic number density of this layer by simply dividing the SLD of the virgin layer, $S L D_{M g}$ by $b_{M g}$.
If one assumes that the number of $\mathrm{Mg}$ atoms remains constant within the layer, one can compute the number density of the deuterated layer with an unknown $x$ from

$$
N_{M g D_{X}}=N_{M g} \frac{d_{M g}}{d_{M g D_{X}}}=\frac{S L D_{M g}}{b_{M g}} \frac{d_{M g}}{d_{M g D_{X}}},
$$

where $d_{i}$ denotes the thickness of layer $i$. Substituting this expression in equation (1) and isolating $x$ provides

$$
x=\left(\frac{S L D_{M g D_{X}}}{S L D_{M g}} \frac{d_{M g D_{X}}}{d_{M g}}-1\right) \frac{b_{M g}}{b_{D}} .
$$

\section{Two-phase model}

When the domain sizes during loading exceed the coherence length of the neutron, estimated to be around 30 $\mu \mathrm{m}$, the measured neutron reflectogram becomes a superposition of the reflectograms of two different contributions: one of the $\alpha-\mathrm{MgD}_{\mathrm{x}}$ phase and one of the $\beta-\mathrm{MgD}_{2-\mathrm{y}}$ phase. Hence, it becomes possible to determine the scattering length density and layer thickness of the different layers of the phases separately. 
The two-phase model is illustrated with an example of a timeslice at $t=780-810 \mathrm{~min}$ as depicted in Figure $\mathbf{S 2}$. We simultaneously fit two models, one for the $\alpha-\mathrm{MgD}_{\mathrm{x}}$ phase $R_{\alpha}\left(Q_{z}\right)$ and one of the $\beta-\mathrm{MgD}_{2-\mathrm{y}}$ phase $R_{\beta}\left(Q_{z}\right)$. Both of these models use the same fitting values for the titanium layer thickness and SLD to keep the number of parameters that are fitted to a minimum. Subsequently, the two models superimposed with their relative contribution to obtain the combined reflectogram

$$
R\left(Q_{z}\right)=(1-f) R_{\alpha}\left(Q_{z}\right)+f R_{\beta}\left(Q_{z}\right),
$$

where $f$ is the fraction of $\beta-\mathrm{MgD}_{2-y}$ that is determined from the hydrogenography measurements. This fraction is equal to $f=0.78$ in the example given.

To illustrate that the two-phase model is the correct way to fit this data, we also fitted the $t=780-810$ min time slice with a model that assumes that the Mg layer is composed of one phase and depicted in Figure $\boldsymbol{S}_{\mathbf{3}}$. The figure shows that the one-phase fit does not properly describe the measured data.

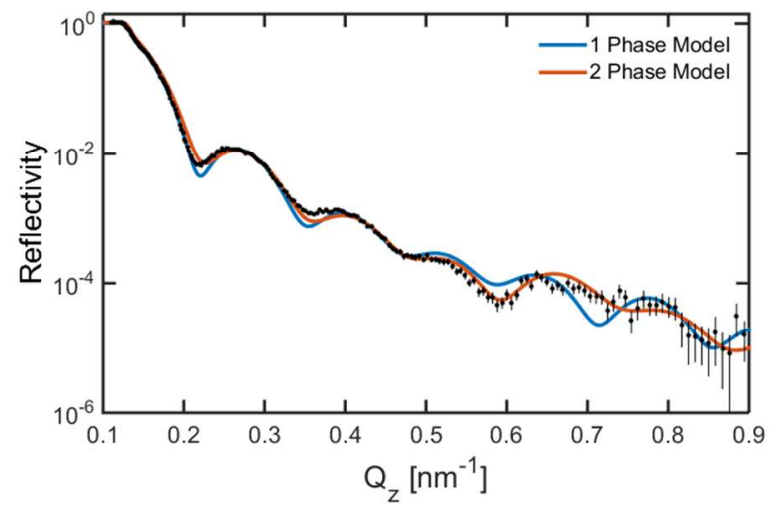

Fig. $S_{3}$ Comparison of the performance 1-phase model and the 2-phase model for time slice $t=780-810 \mathrm{~min}$.

Table S1 Fitted parameters of selected reflection curves at various instances during the second loading. The o min time slice refers to the situation after the first unloading at $P_{D_{2}}=4 \mathrm{~Pa}$.

\begin{tabular}{|c|c|c|c|c|c|c|c|c|}
\hline \multirow[t]{2}{*}{ Loading Time (min) } & \multirow[t]{2}{*}{ Virgin } & \multirow[t]{2}{*}{$\mathbf{o}$} & \multirow[t]{2}{*}{5} & \multirow[t]{2}{*}{420} & \multicolumn{2}{|c|}{780} & \multirow[t]{2}{*}{900} & \multirow[t]{2}{*}{1200} \\
\hline & & & & & $\alpha$-phase & $\beta$-phase & & \\
\hline$\sigma_{\mathrm{amb}}(\mathrm{nm})$ & 0.98 & 2.00 & 2.00 & 2.00 & 1.65 & 1.65 & 1.57 & 1.19 \\
\hline \multicolumn{9}{|c|}{ Pd-Layer } \\
\hline$d(\mathrm{~nm})$ & 9.60 & 9.60 & 9.60 & 9.60 & 9.60 & 9.60 & 9.60 & 9.60 \\
\hline $\operatorname{SLD}\left(10^{-4} \mathrm{~nm}^{-2}\right)$ & 4.02 & 4.02 & 4.02 & 4.02 & 4.02 & 4.02 & 4.02 & 4.02 \\
\hline$\sigma(\mathrm{nm})$ & 1.65 & 3.00 & 1.64 & 0.52 & 1.54 & 1.54 & 1.63 & 2.10 \\
\hline \multicolumn{9}{|c|}{ Ti-Layer (up) } \\
\hline$d(\mathrm{~nm})$ & "'"'mo & 12.49 & $13 \cdot 50$ & 13.50 & 13.03 & 13.03 & 13.23 & 13.5 \\
\hline $\operatorname{SLD}\left(10^{-4} \mathrm{~nm}^{-2}\right)$ & -1.95 & 0.05 & 2.39 & $3 \cdot 45$ & 3.98 & 3.98 & 4.15 & 4.20 \\
\hline$\sigma(\mathrm{nm})$ & 1.41 & 3.00 & 0.01 & 0.02 & 0.01 & 0.01 & 0.01 & 0.01 \\
\hline \multicolumn{9}{|c|}{ Mg-Layer } \\
\hline$d(\mathrm{~nm})$ & 9.60 & 9.60 & 9.60 & 11.30 & $9 \cdot 57$ & 13.52 & $13 \cdot 32$ & $13 \cdot 52$ \\
\hline $\operatorname{SLD}\left(10^{-4} \mathrm{~nm}^{-2}\right)$ & 2.00 & 2.12 & 2.44 & 2.71 & 2.98 & 4.62 & $4 \cdot 52$ & 4.80 \\
\hline$\sigma(\mathrm{nm})$ & 1.40 & 2.95 & 0.00 & 0.00 & 0.14 & 0.00 & 0.14 & 0.00 \\
\hline \multicolumn{9}{|c|}{ Ti-Layer (down) } \\
\hline$d(\mathrm{~nm})$ & 9.88 & $9 \cdot 40$ & 8.45 & 10.20 & 10.60 & 10.60 & 8.11 & 9.83 \\
\hline $\operatorname{SLD}\left(10^{-4} \mathrm{~nm}^{-2}\right)$ & -1.95 & -0.89 & 2.09 & 3.23 & $3 \cdot 75$ & 2.75 & 3.63 & $3 \cdot 72$ \\
\hline$\sigma(\mathrm{nm})$ & 0.13 & 2.86 & 3.00 & 0.00 & 1.36 & 1.36 & 1.98 & 0.02 \\
\hline \multicolumn{9}{|c|}{ Ti-Layer (down) (Depletion Layer) } \\
\hline$d(\mathrm{~nm})$ & "'meo & "3.'" & 3.00 & 1.33 & 2.34 & 2.34 & 1.61 & 0.77 \\
\hline $\operatorname{SLD}\left(10^{-4} \mathrm{~nm}^{-2}\right)$ & 0.00 & -0.59 & 2.21 & 1.11 & 1.77 & 1.77 & 2.89 & 2.65 \\
\hline$\sigma(\mathrm{nm})$ & 0.00 & 1.92 & 1.54 & 0.01 & 0.14 & 0.14 & 2.37 & 2.75 \\
\hline \multicolumn{9}{|c|}{ Substrate } \\
\hline $\operatorname{SLD}\left(10^{-4} \mathrm{~nm}^{-2}\right)$ & 3.42 & $3 \cdot 42$ & 3.42 & 3.42 & 3.42 & 3.42 & 3.42 & $3 \cdot 42$ \\
\hline
\end{tabular}


Table $S_{2}$ Fitted parameters of selected reflection curves at various instances during the second unloading at $\boldsymbol{P}_{D_{2}}$ $=10 \mathrm{~Pa}$.

\begin{tabular}{|c|c|c|c|c|c|c|}
\hline Unloading Time (min) & Virgin & Loaded & 25 & 65 & 105 & 255 \\
\hline$\sigma_{\mathrm{amb}}(\mathrm{nm})$ & 0.98 & 1.19 & 2.00 & 2.00 & 1.65 & 1.65 \\
\hline \multicolumn{7}{|c|}{ Pd-Layer } \\
\hline$d(\mathrm{~nm})$ & 9.60 & 9.60 & 9.60 & 9.60 & 9.60 & 9.60 \\
\hline $\operatorname{SLD}\left(10^{-4} \mathrm{~nm}^{-2}\right)$ & 4.02 & 4.02 & 4.02 & 4.02 & 4.02 & 4.02 \\
\hline$\sigma(\mathrm{nm})$ & 1.65 & 2.10 & 291 & 2.69 & 2.69 & 2.62 \\
\hline \multicolumn{7}{|c|}{ Ti-Layer (up) } \\
\hline$d(\mathrm{~nm})$ & 9.80 & $13 \cdot 5$ & $13 \cdot 50$ & 13.50 & 13.03 & 13.03 \\
\hline $\operatorname{SLD}\left(10^{-4} \mathrm{~nm}^{-2}\right)$ & -1.95 & 4.20 & 3.81 & 3.48 & $3 \cdot 31$ & 3.22 \\
\hline$\sigma(\mathrm{nm})$ & 1.41 & 0.01 & 0.01 & 0.05 & 0.01 & 0.01 \\
\hline \multicolumn{7}{|c|}{ Mg-Layer } \\
\hline$d(\mathrm{~nm})$ & 9.60 & $13 \cdot 5^{2}$ & 13.12 & 11.99 & 10.46 & 10.06 \\
\hline $\operatorname{SLD}\left(10^{-4} \mathrm{~nm}^{-2}\right)$ & 2.00 & 4.80 & $4 \cdot 35$ & 3.13 & 2.65 & 2.44 \\
\hline$\sigma(\mathrm{nm})$ & 1.40 & 0.00 & 0.03 & 0.08 & 0.14 & 0.01 \\
\hline \multicolumn{7}{|c|}{ Ti-Layer (down) } \\
\hline$d(\mathrm{~nm})$ & 9.88 & 9.83 & 10.47 & 9.06 & $9 \cdot 31$ & 9.98 \\
\hline $\operatorname{SLD}\left(10^{-4} \mathrm{~nm}^{-2}\right)$ & -1.95 & 3.72 & $3 \cdot 56$ & 3.35 & $3 \cdot 35$ & 3.24 \\
\hline$\sigma(\mathrm{nm})$ & 0.13 & 0.02 & 1.74 & 2.99 & 3.00 & 3.01 \\
\hline \multicolumn{7}{|c|}{ Ti-Layer (down) (Depletion Layer) } \\
\hline$d(\mathrm{~nm})$ & 0.00 & 0.77 & 1.15 & 1.43 & 2.99 & 2.99 \\
\hline $\operatorname{SLD}\left(10^{-4} \mathrm{~nm}^{-2}\right)$ & o.oo & 2.65 & 0.51 & 3.87 & 2.58 & 2.81 \\
\hline$\sigma(\mathrm{nm})$ & 0.00 & 2.75 & 2.74 & 2.26 & 2.27 & 0.11 \\
\hline \multicolumn{7}{|c|}{ Substrate } \\
\hline $\operatorname{SLD}\left(10^{-4} \mathrm{~nm}^{-2}\right)$ & 3.42 & 3.42 & 3.42 & 3.42 & 3.42 & 3.42 \\
\hline
\end{tabular}


(a)

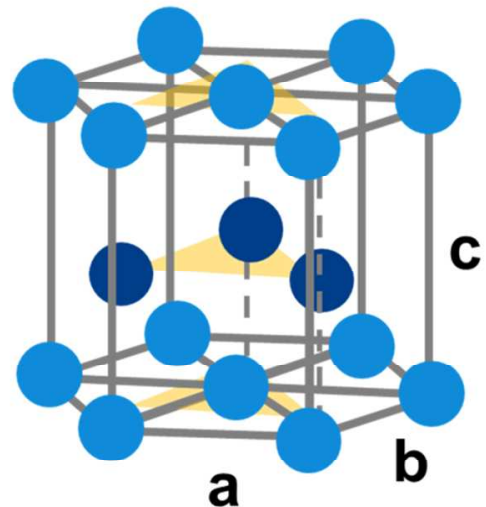

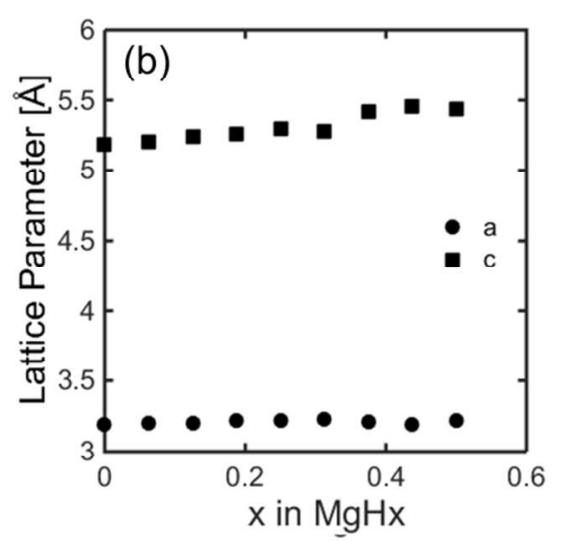

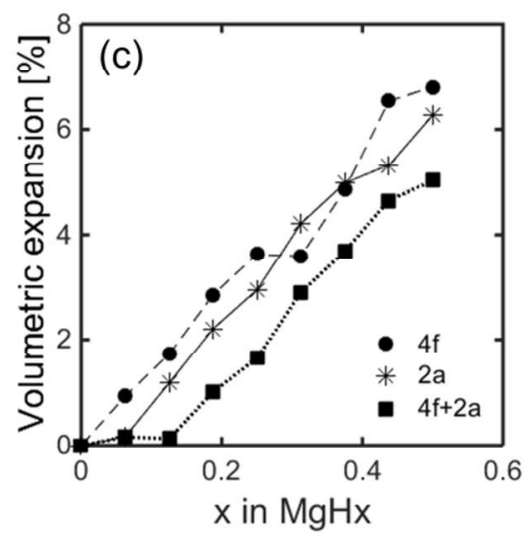

Figure S4. (a) Schematic representation of the Hexagonal Close Packed (HCP) $\alpha$-Mg crystal structure (194-P63/mmc) with lattice cell parameters $a=0.321, b=0.321, c=0.523 \mathrm{~nm}$. (b) Lattice parameter as a function of $x$ in $\mathrm{MgH}_{\mathrm{x}}$ where the hydrogen atoms have been inserted in the $4 \mathrm{f}$ sites $(1 / 3,2 / 3,0.08)$ only. The expansion of the $b$-axis as a function of the number of inserted hydrogen atoms is similar to the expansion of the a-axis. (c) Volumetric expansion of the $\alpha$-Mg unit cell where the hydrogen atoms have been inserted at the $4 \mathrm{f}(1 / 3,2 / 3,0.08), 2 \mathrm{a}(0,0,0)$ or at both the $4 \mathrm{f}$ and $2 \mathrm{a}$ sites.

\section{DFT Calculations - Method}

Density Functional Theory (DFT) calculations are performed with the Vienna Ab-initio Simulation Package (VASP) to acquire a better understanding of the effect of the insertion and removal of deuterium from the $\alpha$ $\mathrm{MgH}_{\mathrm{x}} / \beta-\mathrm{Mg}$ optical properties of the unit cell. ${ }^{34}$ For the potentials of the atoms, the Generalized Gradient Approximation (GGA) is used..$^{35}$ Brillouin zone integrations are performed with the help of the Monkhorst-Pack 8x8x8 kpoint mesh for the $\alpha-\mathrm{Mg}$ and a $5 \times 5 \times 5 \mathrm{k}$-point mesh for the $\beta$-Mg structure. ${ }^{36} \mathrm{~A} 2 \mathrm{2} 2 \times 2$ super-cell with sixteen magnesium atoms is used and the unit cell parameters are allowed to relax freely.

For the calculations of hydrogen in the hexagonal close packed $\alpha-\mathrm{Mg}$ crystal structure $(194-\mathrm{P} 63 / \mathrm{mmc})$ with unit cell parameters $a=0.321, b=0.321, c=0.523 \mathrm{~nm}$ as schematically depicted in Figure $\mathbf{S}_{\mathbf{4}}$-a, hydrogen atoms are inserted at both the $4 \mathrm{f}(1 / 3,2 / 3,0.08)$ and $2 \mathrm{a}(0,0,0)$ sites. These sites have been selected based on Molecular Dynamics computations of Schimmel et al. and as previously done by Mulder et al. ${ }^{30,12}$ We inserted the hydrogen atoms in 5 different configurations, both at the za sites only, the $4 \mathrm{f}$ sites only and in three possible ways in both the $2 \mathrm{a}$ and $4 \mathrm{f}$ sites. We realize that the possible unit cells considered is only a small part of the total number of possible options, especially when more hydrogen atoms are inserted. However, we believe that the results can be used to gain insight in the electronic properties of the material, i.e. whether it stays metallic or becomes insulating. For the calculations, as generally used for metals, the MethfesselPaxton scheme of order one is used. This choice has been checked for robustness purposes but was not found to influence the results significantly.

Calculations for the insulating $\beta-\mathrm{Mg}$ hydride structure $\left(136-\mathrm{P}_{42} / \mathrm{mnm}\right)$ with unit cell parameters $a=0.451, b=$ $0.451, c=0.301 \mathrm{~nm}$ have been performed. Both a Gaussian and a Methfessel-Paxton scheme of order one have been used. To obtain the density of states, first a relaxation has been performed, and subsequently the density of states has been computed. To compute the band structure, the relaxation was performed using a three dimensional $\mathrm{k}$ mesh, and was subsequently computed at and between symmetry points in the Brillouin zone.

\section{DFT Calculations - Results}

Figure $\mathrm{S}_{4}$-b displays the obtained unit lattice parameters of the unit cell for the insertion of different quantities of hydrogen atoms at the $4 \mathrm{f}$ sites in $\alpha-\mathrm{MgH}_{\mathrm{x}}$. The computed value for the lattice parameters of $\alpha-\mathrm{MgH}_{0.0}$ of $a=$ $0.319, b=0.319, c=0.518$ are in good agreement with the experimental reported values of $a=0.321, b=0.321, c=$ $0.523 .{ }^{36}$ The insertion of hydrogen atoms results mostly in an expansion of the $c$-axis. When hydrogen atoms are inserted beyond $\mathrm{MgH}_{0.4}$, the angles between the axes start to deviate up till $10^{\circ}$ from their values for $\alpha-\mathrm{MgH}_{0.0}$ 'The relation between the volumetric expansion and the amount of hydrogen inserted (Figure S1-c) in the unit cell appears linear for all considered locations of insertion of the hydrogen atoms. A hydrogen content of $\mathrm{MgH}_{0.4}$ results in a modest expansion of approximately $5 \%$.

The computed lattice parameters of the $\beta-\mathrm{MgH}_{2}$ hydride structure as schematically displayed in Figure $\mathbf{S}_{\mathbf{5}}$-a are $a=0.449, b=0.449, c=0.301 \mathrm{~nm}$ and are in excellent agreement with experimental obtained values reported in literature of $a=0.451, b=0.451, c=0.301 \mathrm{~nm} .{ }^{37}$ The removal of hydrogen atoms from the $\beta-\mathrm{Mg}$ hydride structure results mainly in a decrease of the $b$-axis of the unit cell (Figure $\mathrm{S}_{5}-\mathrm{b}$ ). This decrease increases when hydrogen atoms are removed beyond $\mathrm{MgH}_{1.8}$. The volumetric expansion with respect to the volume of the $\alpha-\mathrm{Mg}$ unit cell displayed in Figure $\mathrm{S}_{5}$-c shows a similar trend.

The computed band structure and density of states for $\mathrm{MgH}_{2}$ is depicted in Figure $\mathrm{S}_{5}-\mathrm{d}$. The obtained value for 
(a)

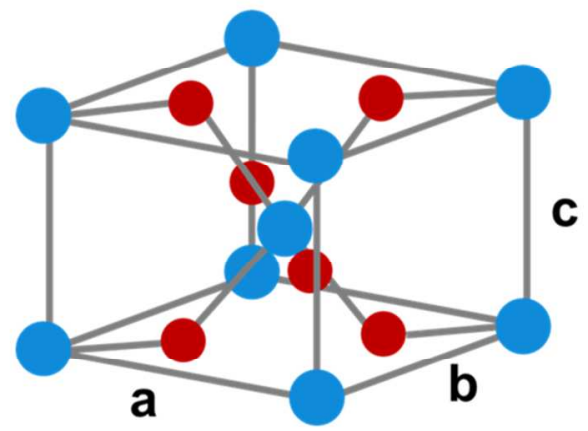

(d)

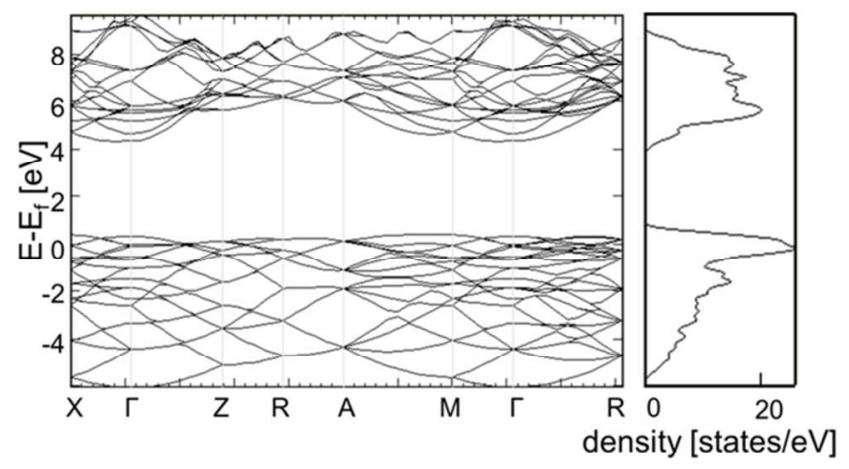

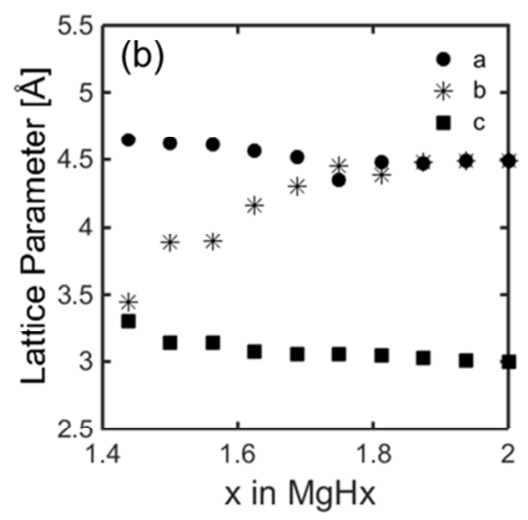
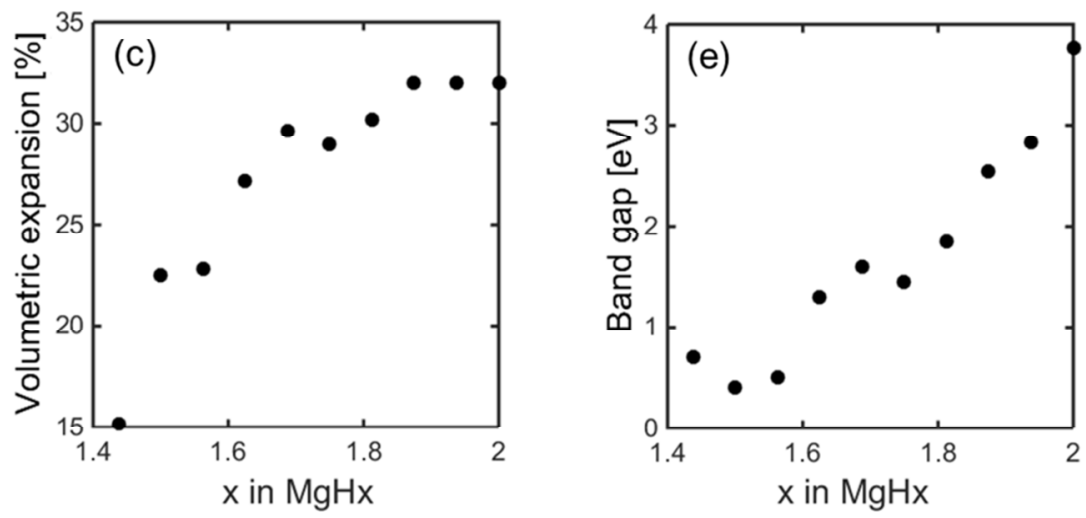

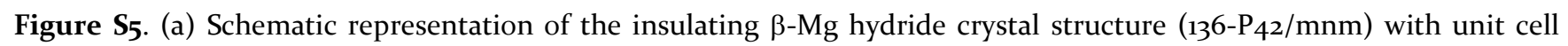
parameters $a=0.451, b=0.451, c=0.301 \mathrm{~nm}$. The red spheres represent the hydrogen atoms and the blue spheres the magnesium atoms. (b) Lattice parameter as a function of $x$ in $\mathrm{MgH}_{\mathrm{x}}$ where the hydrogen atoms have been removed from the $\beta-\mathrm{MgH}_{2}$ crystal. (c) Volumetric expansion of the $\beta$ - Mg unit cell for various amounts of hydrogen atoms removed from the unit cell. (d) Computed band structure and density of states for $\mathrm{MgH}_{2}$. (e) Evolution of the band gap as a function of the amount of hydrogen in $\beta-\mathrm{Mg}$.

the band gap of $\mathrm{MgH}_{2}$ is $3.9 \mathrm{eV}$, lower than the experimentally observed band gap of $5.6 \pm 0.1 \mathrm{eV}$ but in good agreement with previous DFT studies on $\mathrm{MgH}_{2}$ by e.g. Flacau et al. who also found a band gap of $3.9 \mathrm{eV} \cdot{ }^{39}$ This underestimation of the band gap is a known deviation of GGA-DFT, but as we are only interested in qualitative computations, we believe that this does not limit the applicability of the computations for the present study. ${ }^{29}$

The band gap is found to decrease when an increasing amount of hydrogen atoms are removed from the $\beta-\mathrm{MgH}_{2}$ unit cell (Figure $\mathrm{S}_{5}-\mathrm{e}$ ). We find that at least a band gap of $1.5 \mathrm{eV}$ exists until $\mathrm{MgH}_{1.6}$. This is slightly lower than the peak intensity of our light source $(2.0 \mathrm{eV})$, however, due to the method used, the computed band gap is likely to be an underestimation.

\section{REFERENCES}

(32) Dalgliesh, R.; Langridge, S.; Plomp, J.; De Haan, V.; Van Well, A. Offspec, the ISIS Spin-Echo Reflectometer. Physica B 2011, 406, 2346-2349.

(33) Van de Kruijs, R. Specular and Off-Specular Reflection of Polarized Neutrons from Magnetic Thin Films and Multilayers, PhD thesis, Delft University of Technology, 2002.
(34) Kresse, G.; Furthmüller, J. Efficient Iterative Schemes for $a b$ initio Total-Energy Calculations Using a Plane-Wave Basis Set. Phys. Rev. B 1996, 54, 11169-11186.

(35) Perdew, J.; Zunger, A. Self-Interaction Correction to Density-Functional Approximations for Many-Electron Systems. Phys. Rev. B 1981, 23, 5048-5079.

(36) Monkhorst, H.; Pack, Special Points for Brillouin-Zone Integrations J. Phys. Rev.B 1976, 13, 5188-5192.

(37) Takasaki, T.; Kyoi, D.; Kitamura, N.; Tanase, S; Sakai, T. Reversible Hydrogen Storage Property and Structural Analysis for Face-Centered Cubic Hydride $\mathrm{Mg}_{0.82} \mathrm{Zr}_{\mathrm{o} .18} \mathrm{H}_{2}$ Prepared by Gigapascal Hydrogen Pressure Method. J. Phys. Chem. B, 2007, 111, 14102-1406.

(38) Takeichi, N.; Shida, K.; Yang, X.; Sakai, T. Crystal Structure and Hydrogen Storage Properties of Novel Mg-Zr-(Li, Na, K) Hydrides Prepared by Gigapascal Hydrogen Pressure Method, MRS Proceedings. 2011, 1334, 1502.

(39)Kelkar, T.; Pal, S.; Kanhere, G. Density Functional Investigations of Electronic Structure and Dehydrogenation Reactions of Al- and Si-Substituted Magnesium Hydride. ChemPhysChem 2008, 9, 928-934. 